\title{
IMF dependence of high-latitude thermospheric wind pattern derived from CHAMP cross-track measurements
}

\author{
M. Förster ${ }^{1}$, S. Rentz ${ }^{1}$, W. Köhler ${ }^{1}$, H. Liu ${ }^{2}$, and S. E. Haaland ${ }^{3,4}$ \\ ${ }^{1}$ GeoForschungsZentrum (GFZ) Potsdam, Potsdam, Germany \\ ${ }^{2}$ Hokkaido University, Div. of Earth and Planet. Science, Sapporo, Japan \\ ${ }^{3}$ Max-Planck Institute für extraterrestrische Physik (MPE), Garching, Germany \\ ${ }^{4}$ Department of Physics, University of Bergen, Norway
}

Received: 13 December 2007 - Revised: 7 April 2008 - Accepted: 16 April 2008 - Published: 11 June 2008

\begin{abstract}
Neutral thermospheric wind pattern at high latitudes obtained from cross-track acceleration measurements of the CHAMP satellite above both North and South polar regions are statistically analyzed in their dependence on the Interplanetary Magnetic Field (IMF) direction in the GSM $\mathrm{y}-\mathrm{z}$ plane (clock angle). We compare this dependency with magnetospheric convection pattern obtained from the Cluster EDI plasma drift measurements under the same sorting conditions. The IMF-dependency shows some similarity with the corresponding high-latitude plasma convection insofar that the larger-scale convection cells, in particular the round-shaped dusk cell for $B_{y}^{\mathrm{IMF}}+\left(B_{y}^{\mathrm{IMF}}-\right)$ conditions at the Northern (Southern) Hemisphere, leave their marks on the dominant general transpolar wind circulation from the dayside to the nightside. The direction of the transpolar circulation is generally deflected toward a duskward flow, in particular in the evening to nighttime sector. The degree of deflection correlates with the IMF clock angle. It is larger for $B_{y}^{\mathrm{IMF}}+$ than for $B_{y}^{\mathrm{IMF}}-$ and is systematically larger $\left(\sim 5^{\circ}\right)$ and appear less structured at the Southern Hemisphere compared with the Northern. Thermospheric cross-polar wind amplitudes are largest for $B_{z}^{\mathrm{IMF}}-/ B_{y}^{\mathrm{IMF}}-$ conditions at the Northern Hemisphere, but for $B_{z}^{\mathrm{IMF}}-/ B_{y}^{\mathrm{IMF}}+$ conditions at the Southern because the magnetospheric convection is in favour of largest wind accelerations over the polar cap under these conditions. The overall variance of the thermospheric wind magnitude at Southern high latitudes is larger than for the Northern. This is probably due to a larger "stirring effect" at the Southern Hemisphere because of the larger distance between the geographic and geomagnetic frameworks.
\end{abstract}

Keywords. Magnetospheric physics (Plasma convection; Polar cap phenomena) - Meteorology and atmospheric dynamics (Thermospheric dynamics)

Correspondence to: $\mathrm{M}$. Förster

(mfo@gfz-potsdam.de)

\section{Introduction}

Thermospheric winds respond rather directly via ion-drag to ionospheric plasma motions that are imposed by the solar wind-magnetosphere interaction and the resulting largescale magnetospheric plasma drift at high latitudes. The largest magnitudes of neutral wind speed anywhere on the globe were found to occur within the high-latitude upper thermosphere. Long-term observations with groundbased Fabry-Perot interferometers (FPI) located at Thule and Søndre Strømfjord, Greenland, for example, showed typical wind speeds of about $200 \mathrm{~m} / \mathrm{s}$ at solar minimum, rising up to about $800 \mathrm{~m} / \mathrm{s}$ at solar maximum, depending on geomagnetic activity level (Killeen et al., 1995).

Subject to the local plasma density, the response of the high-latitude thermosphere is not immediate, but is rather measured in tens of minutes to hours (Ponthieu et al., 1988). The inertia of the thermospheric neutral winds, on the other hand, can help to maintain the ionospheric convection independently of the magnetospheric driver processes. This has been known for long time as the fly-wheel effect (Banks, 1972; Coroniti and Kennel, 1973).

Relative motions of the neutral gas and ionized components result in Joule heating of the ionosphere and thermosphere, being proportional to the square of the velocity difference. A recent study showed mesoscale structures and variations of the thermosphere on the scales of tens of kilometers and minutes that indicate a very dynamic response to ionospheric forcing (Aruliah et al., 2004). The irregular part of the relative motions have been shown to be an essential contributor of the energy budget. This is mainly due to the large variability of the high-latitude convection which interacts with the neutral gas of the upper thermosphere where it is embedded (Codrescu et al., 1995, 2000; Crowley and Hackert, 2001; Matsuo et al., 2003; Förster et al., 2007).

For the usual antisunward transpolar flow within the central polar cap (near the geographic pole), the centrifugal force

Published by Copernicus Publications on behalf of the European Geosciences Union. 
vanishes, while for the off-center antisunward flow within the polar cap the Coriolis and centrifugal forces act differently on the dawn and dusk side. On the dawn side (westward flow) they have the tendency to cancel each other, while on the dusk side (eastward flow) they add to form a stronger force for duskward deflection of the air flow. At lower, subauroral latitudes, where a sunward flow could evolve, the opposite interference of the acceleration terms would be realized.

Numerous early studies of the cross-polar wind circulation and its response to Interplanetary Magnetic Field (IMF) conditions were mainly based on satellite observations of Dynamics Explorer 2 (DE-2) and Atmospheric Explorer-C (AE-C) (Killeen et al., 1984, 1985; McCormac et al., 1985; Thayer et al., 1987; Ponthieu et al., 1988), or on combined satellite and various ground-based FPI measurements (Hays et al., 1979; Killeen et al., 1986, 1995). Although these satellite studies had to rely on a quite limited statistical basis, they came in combination with numerical studies using thermospheric general circulation models (TGCMs) to remarkable insights about the IMF dependencies of the high-latitude neutral wind pattern (e.g. Hays et al., 1984; Killeen and Roble, 1984; Rees et al., 1986).

More recent studies come mainly from ground-based observations. They are restricted therefore to a few selected observatories at high latitudes but comprise long time series as, e.g. the studies of Aruliah et al. (1996), Griffin et al. (2004a,b), and Emmert et al. (2006a,b). Satellite missions on this topic were quite rare during the last two decades, with the exception of the Wind Imaging Interferometer (WINDII) (Shepherd et al., 1993) onboard the Upper Atmosphere Research Satellite (UARS) and the Doppler Interferometer (TIDI FPI) onboard the TIMED satellite (Killeen et al., 2006). The orbit of UARS was not well suited for polar latitudes, nevertheless Richmond et al. (2003) could statistically analyze IMF dependencies of high-latitude lower thermosphere wind pattern at various height levels between $105 \mathrm{~km}$ and $300 \mathrm{~km}$. Together with the newly-established ground-based FPIs, most new insights into the high-latitude neutral wind structure during the last two decades were achieved for altitudes well below $300 \mathrm{~km}$ mainly for the mesosphere and lower thermosphere.

The highly sensitive accelerometer onboard CHAMP at its polar orbit at F-region height, that is used for this study, allows the measurement of the cross-track neutral wind on a global scale (Liu et al., 2006). The use of accelerometers to measure thermospheric winds have been reported quite rarely (Marcos and Forbes, 1985; Forbes et al., 1993). The advantage of this technique consists in the quite direct in-situ measurement along the orbital track with only a limited number of special assumptions for the data interpretation. Their more ample use at other satellites (e.g. GRACE) and for forthcoming missions, will allow better statistical studies of the upper thermosphere dynamics in near future.

In two previous papers (Haaland et al., 2007; Förster et al., 2007), statistical patterns of the high-latitude magnetospheric convection electric field and their relation to solar wind conditions, in particular the IMF dependency were presented. They have been inferred from electron drift measurements of the Electron Drift Instruments (EDI) onboard the Cluster satellites during the years 2001-2006.

Here we are interested particularly in the thermospheric response to the driving forces of the large-scale magnetospheric convection that results from solar wind and IMF interactions with the magnetosphere. We sort therefore the observations with respect to high-latitude geomagnetic coordinates. Due to large differences in the geomagnetic field configuration and a clear difference in the offsets between the geographic and geomagnetic poles at the North and South Hemisphere, we expect a different degree of obedience of the neutral gas to the plasma drift between the two opposite polar regions. As will be shown in the succeeding sections, there is an interference of the geomagnetic field on the transpolar circulation.

\section{The data}

\subsection{CHAMP dataset}

The challenging minisatellite payload (CHAMP) is managed by the GeoForschungsZentrum (GFZ) Potsdam. This mission is designed to perform detailed studies of the Earth's gravitational and magnetic field with unprecedented accuracies and space/time resolutions as well as GPS atmosphere and ionosphere profiling. The spacecraft was launched in July 2000 into a circular near-polar orbit with $87.3^{\circ}$ inclination at an altitude of $\sim 460 \mathrm{~km}$ (Reigber et al., 2002). Its orbital altitude gradually decayed and is $\sim 400 \mathrm{~km}$ in 2003 .

One key scientific instrument onboard CHAMP is a triaxial accelerometer. It is located at the spacecraft's center of mass and effectively probes the in situ air drag with an accuracy of $1 \times 10^{-14} \mathrm{~kg} \mathrm{~m}^{-3}$ (Liu et al., 2005). From the air drag observations, thermospheric mass density and crosstrack neutral wind can be obtained using the methodology described in Liu et al. (2006). Using these cross-track wind measurements, the high-latitude thermospheric wind patterns are constructed in this study.

As pointed out by Liu et al. (2006), the along-track wind is very difficult to measure due to its relatively small magnitude in comparison to the satellite speed of $7.6 \mathrm{~km} \mathrm{~s}^{-1}$. Therefore, the derivation of the cross-track wind neglects the effect of the along-track wind. This neglection causes little error in the cross-track wind at low and middle latitudes due to the small magnitude of the meridional wind. However, in polar areas the along-track (meridional) wind velocity, as will be shown with the wind pattern derived below, can achieve about $10 \%$ of the satellite speed. Consequently, it can cause an error of about $10 \%$ in the cross-track wind in the polar region.

The errors due to the instrument's precision (about $3 \times 10^{-9} \mathrm{~m} \mathrm{~s}^{-2}$ ) with $\sim 20 \mathrm{~m} \mathrm{~s}^{-1}$ and due to systematic 
contributions from other sources (see Liu et al., 2006, and the error budget in Appendix A of that paper) with $\sim 15 \mathrm{~m} \mathrm{~s}^{-1}$ are relatively small and negligible compared to this uncertainty. The pre-processed data of the accelerometer were re-sampled to 10-s averages for the further use in this study. Measurements of 10-s cadence correspond to a spatial separation of $76 \mathrm{~km}$ or about $2 / 3 \mathrm{deg}$ in latitude between the individual data points.

The precessing rate of CHAMP's orbital plane is $15^{\circ}$ within an 11-day period. Considering the two wings of an orbit at opposite local times, a full coverage of all local times is achieved within about 131 days. In this study we use a time interval of nearly the three-fold of this length, the full year 2003, during which each local time is scanned by both ascending and descending orbital paths. This way we obtain about an equal data coverage at both hemispheres for all seasons. The solar activity, as expressed by the $\mathrm{F}_{10.7}$ index, is at a moderate level of about 130 during this interval, but the geomagnetic activity was very high throughout this year of the declining solar activity cycle. In an earlier study on average thermospheric wind pattern over the polar regions (Lühr et al., 2007), a part of this time interval, centered around June solstice, was already used for a study which focused on seasonal differences of the transpolar circulation.

\subsection{Solar wind and IMF data}

In this study, we take great care on reliably well defined concurrent IMF and solar wind conditions for any moment of the CHAMP observations. The IMF and solar wind data are taken from the Advanced Composition Explorer (ACE) spacecraft which is positioned near the L1 libration point about $200 R_{E}$ upstream of Earth. We have used data from the magnetic field instrument MAG (Smith et al., 1998) at 16-s resolution and the solar wind instrument SWEPAM (McComas et al., 1998) at 64-s resolution. MAG and SWEPAM data are resampled to one minute time resolution, thereafter time shifted to represent the IMF conditions at the frontside magnetopause (at $X_{\mathrm{GSM}}=10 R_{E}$ ) using the phase front propagation technique (Weimer et al., 2003) in a slightly modified method that is based on a constrained minimum variance analyses of the IMF (Haaland et al., 2006).

This method is used here in the same manner as in the previous studies on high-latitude plasma convection mentioned already in the Introduction (Haaland et al., 2007; Förster et al., 2007). It comprises, e.g. the treatment of missing ACE data, which are linearly interpolated if the gaps are shorter than $10 \mathrm{~min}$. Intervals with longer data gaps are not used at all, but this concerns only a very small fraction $(<1 \%)$ of the MAG data set and about $20 \%$ of the SWEPAM plasma data.

A running 30-min-interval $(+20 \mathrm{~min} /-10 \mathrm{~min}$ around the actual time) of the time-shifted 1-min MAG data is used to determine the so-called IMF bias vector. The bias vector is calculated from the 31 IMF vectors of this interval in the following way. The projection of the IMF vectors in the GSM $y$-z-plane are first normalized and then the sum of these unit vectors divided by their number is calculated. The resultant vector, the bias vector, constitutes the average clock angle for this interval while its magnitude (between 0 and 1) is a measure for the steady pointing or variability of the IMF (cf. Haaland et al., 2007, Sect. 3.2).

We have done several test runs with various bias vector filterings, that means we selected only data points of intervals where the bias vector magnitude was above a certain threshold. This procedure filters out intervals where the IMF directions change abruptly or where they vary over a larger range. An optimal choice that we used was a threshold of 0.9 , but it appeared that the results were nearly the same as for unfiltered data, while the statistics became poorer (about $45 \%$ of the data are filtered out for this threshold). The plots shown in this paper are therefore done with unfiltered data only. We will note explicitly, where the bias vector filtering was applied for the other statistical analyses. The fact that these are quite insensitive for such filterings means that the thermospheric circulation pattern is relatively inert against rapid changes of the IMF conditions, at least with respect to the 30-min interval chosen for the bias vector construction.

\section{Method}

The CHAMP triaxial accelerometer measurements do not allow the deduction of the full wind vector at once, but only the cross-track wind component, i.e. the y-component within the spacecraft coordinate system where $\mathrm{x}$ points along the satellite orbit, $\mathrm{z}$ toward the Nadir direction, and y complements the right-hand system. To get full horizontal wind pattern over the high-latitude circumpolar regions, we have to rely therefore on a statistical approach. For a particular point in space we combine measurements from different orbits that cross this point at various distinct time moments which are distributed over the whole time interval of measurements in 2003. The reconstruction of the full horizontal wind vector at this point is done with a Singular Value Decomposition (SVD) method which combines the $\mathrm{n}$ individual component measurements $V_{y i}$ of a given bin with their known directions $\boldsymbol{k}_{i}$ in the horizontal plane to a best-fit wind vector $\boldsymbol{V}$ estimation at the bin location that is obtained by minimizing $\delta f$ in:

$$
\sum_{i=1}^{n}\left(V_{y i}-\mathbf{k}_{i} \boldsymbol{V}\right)^{2}=\delta f
$$

This vector estimation is done for each bin of a 2-D grid independently and for simplicity we used the orthogonal Solar Magnetic (SM) coordinates for this reconstruction, i.e. in a first step we transform the $\mathbf{k}_{i}$ of each individual observation from the geocentric coordinates of the CHAMP orbit information into SM coordinates. For the binning of the data we use magnetic coordinates as explained in the 


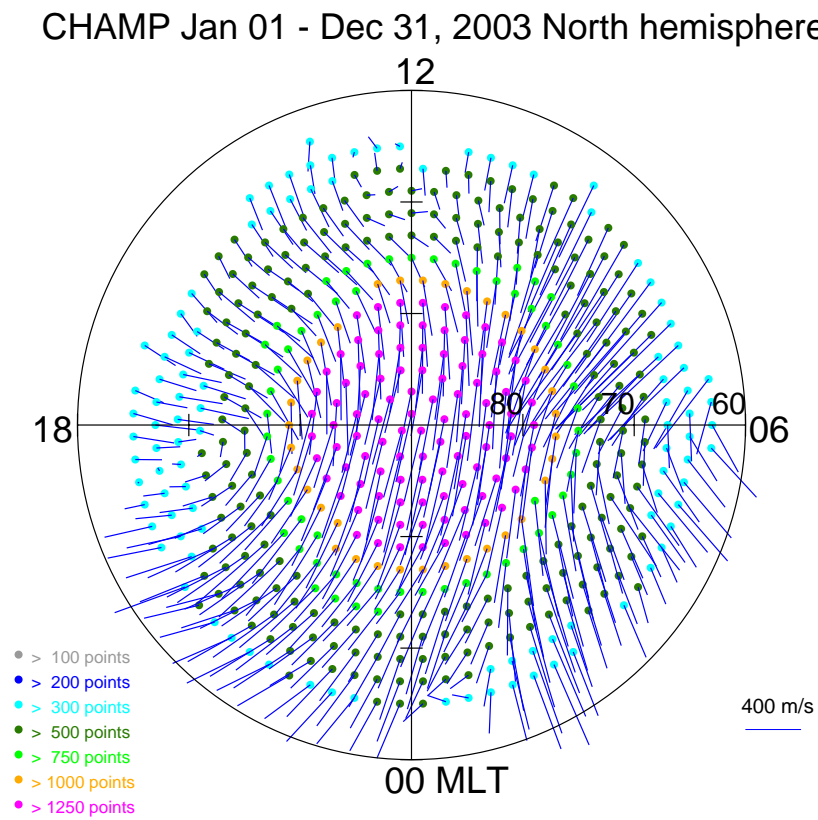

CHAMP Jan 01 - Dec 31, 2003 South hemisphere 12



Fig. 1. North (top) and South Hemisphere (bottom) thermospheric wind pattern at thermospheric heights $(\approx 400 \mathrm{~km})$ using the full CHAMP accelerometer data set of 2003 with all IMF sectors together (summary plot). The wind direction is shown by small vectors with the origin in the dots at the bin's position within the MLT versus magnetic latitude plot; their length indicates the magnitude with the scale given on the lower right side. The number of data points in each bin is colour-coded in the dots with the scale at the lower left.

Introduction. The strong geomagnetic control of the highlatitude thermosphere dynamics has been shown since the early satellite observations of thermospheric neutral air mo- tion as, e.g. by Hays et al. (1984); McCormac et al. (1985); Thayer et al. (1987); Killeen et al. (1986); Richmond et al. (2003). We used modified Apex coordinates (Richmond, 1995), divided both polar regions according to Ruohoniemi and Baker (1998, Eq. 1) into 784 equal-area bins of about 2 deg width covering the high latitude $\left(\geq 58^{\circ}\right)$ thermosphere, and sorted with respect to the direction of the IMF, as measured on ACE. The results of this procedure will be presented subsequently.

But first we have to address the issue of correctness and reliability of the method. The statistical approach implies that we have to compromise between sufficient data points and an optimal resolution of different dependencies. There are important differences between Northern and Southern Hemisphere reconstructions as will be shown below. They are important for the best validity range of the method applied, but could also imply differences in the geoeffectiveness of magnetosphere-ionosphere-thermosphere (MIT) coupling processes at the different hemispheres.

Figure 1 displays summary plots of the resultant thermospheric wind pattern without discriminating for various IMF clock angle ranges. They represent the average neutral wind circulation across the polar cap areas for the time interval under study. These average patterns already reveal the peculiarities that are due to the interaction with the plasma movement like the stagnation area near the cusp footpoint, the large transpolar wind magnitudes with some deflection of the wind vectors toward the dusk side, the large clockwise vortex collocated with the dusk convection cell, and the smaller anticlockwise disturbance in the region of the dawn convection cell. These plots are comparable to the previous paper of Lühr et al. (2007), but use a full year of data coverage that includes about an equal seasonal data selection at both hemispheres.

The colour-coding of the bins down to the $60^{\circ}$ of the plots' outer circle indicate the number of data points per bin while the small blue lines with the origin in the center of each bin show the estimated wind direction and magnitude in the geomagnetic latitude versus Magnetic Local Time (MLT) plot. The best coverage is obtained at higher latitudes where the high-inclination orbit results in many orbital track crossings. At the Northern Hemisphere (Fig. 1, upper panel) this best coverage region is well concentrated close around the magnetic North pole and comprises up to 2200 data points per bin near the center and more than 300 data points in the outermost bins, while at the Southern (lower panel) it is more dispersed with numbers between 400 and 1200 . This is due to the clear difference in the offsets between the geographic and geomagnetic poles at the North and South Hemisphere. Based on the International Geomagnetic Reference Field (IGRF) model, the 2003 location of the north magnetic pole is $82.3^{\circ} \mathrm{N}$ and $114.0^{\circ} \mathrm{W}$ and the south magnetic pole is $64.6^{\circ} \mathrm{S}$ and $138.1^{\circ} \mathrm{E}$. The larger displacement at the Southern Hemisphere leads to a larger diurnal "wobbling" of the polar cap area with respect to the geographic coordinates. 



Fig. 2. Thermospheric wind data obtained from cross-track accelerometer measurements of CHAMP during the year 2003, plotted versus the azimuth angle of the sensor's viewing direction in the X-Y (SM) plane. The azimuth angle is measured from the x-axis (12:00 MLT) toward increasing MLT hours. Wind components from the azimuthal range of $-180^{\circ}$ to $0^{\circ}$ are mirrored onto this range. The bin number and position is indicated in the headline; the parameters of the thermospheric wind estimation are given in the lower left corner: magnitude $|V|$ and azimuth of the wind vector, the standard deviation (STDEV) of the bin's sample and the bias vector magnitude which is a measure for the angular distribution of the component observations (see text).

Figure 2 gives an idea of the data distribution within individual bins. The abscissa is restricted to a half-sphere range of the wind azimuth measured from the $\mathrm{x}$-axis (noon meridian) in the direction of increasing MLT hours from $0^{\circ}$ to $180^{\circ}$. Because of the ambiguity of the wind direction, the wind components from the other half-sphere have been mirrored with opposite sign into this interval. The blue stars indicate the individual cross-track wind component measurements $V_{y i}$, drawn in dependence of the azimuth angle in the SM coordinate system. The red dot shows the estimated wind vector $\boldsymbol{V}$ with its amplitude and azimuth given in the left lower corner of each panel; the red dotted line is the projection of the expected component measurement versus azimuth. The scatter of the actual measurements around this red line gives an idea about the variance of the measurements according to Eq. (2),

$\sigma^{2}=\frac{1}{1-n} \sum_{i=1}^{n}\left(V_{y i}-\mathbf{k}_{i} \boldsymbol{V}\right)^{2}$

where the variables are the same as for Eq. (1) including $\boldsymbol{V}$ as the resultant 2-D wind vector for the given bin. The standard deviation (STDEV) indicated in the lower left corner in each panel of Fig. 2 is the square root of this sample variance value, given in the same units $[\mathrm{m} / \mathrm{s}]$ as the wind magnitude. The left-hand panel shows an example from the central part of sector 2 in Fig. 4 below, where the wind component measurements cover nearly all directions homogeneously. The right-hand panel shows an example of the less-favourably covered bins in the outer regions of the same plot. In the extreme case of the outermost bins of the circumpolar distribution, the individual bins comprise only data points of the oppositely directed orbital parts, the ascending and descending orbital tracks which are recorded about 131 days apart and with the sensors pointing in approximately $180^{\circ}$ opposite directions. It is obvious that the estimated wind vector can be incorrect under such unfavourable, ill-defined conditions. Large values of scatter could result in erroneous results under such conditions.

To quantify the applicability of the method, in particular the coverage of the data points over the azimuth range, we use a bias vector calculation in a similar manner as for the estimation of the solar wind steadiness. The unit vectors $\boldsymbol{k}_{i}$ of each measurement in the corresponding bin are averaged (expanding first the vector's range into a full sphere again). The magnitude of the resulting vector (between 0 and 1) gives a measure for the angular coverage; values near zero mean full coverage while values close to 1 indicate ill-posed conditions with a very restricted range of sensor directions. The bias vector magnitudes (indicated in the bottom line of the examples in Fig. 2 as "bias") are shown in Fig. 3 for the Northern (top) and Southern Hemisphere (bottom) of the full data set sampled in geomagnetic coordinates. Obviously there are essential differences between the hemispheres. The Southern high-latitude region has smaller bias values over a 


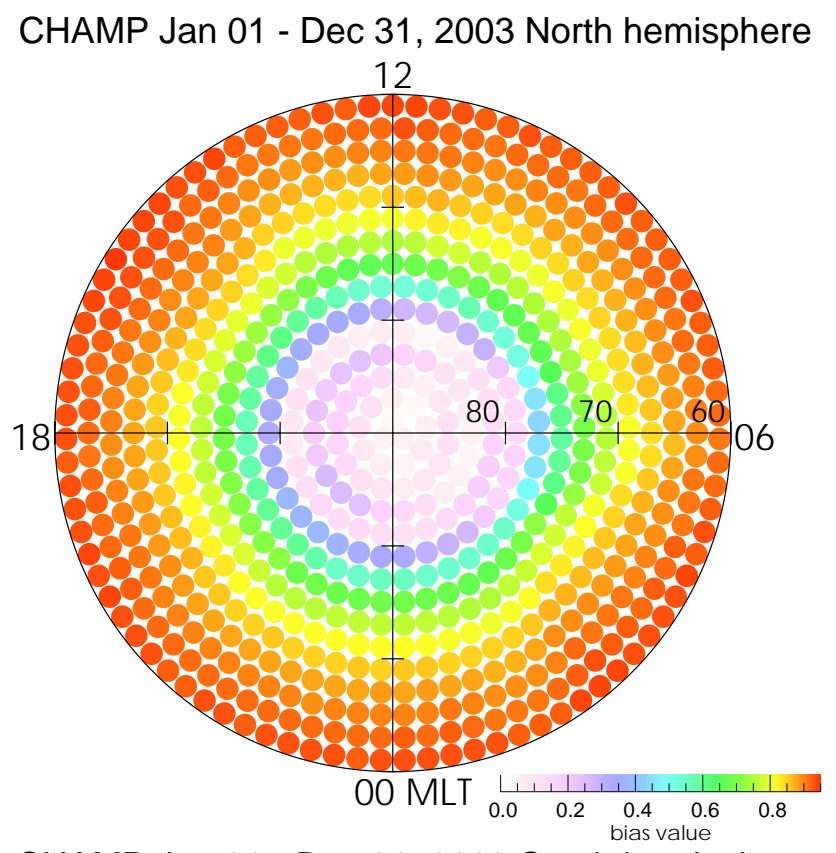

CHAMP Jan 01 - Dec 31, 2003 South hemisphere



Fig. 3. Bias value distribution of the CHAMP cross-track acceleration measurements obtained during the year 2003 over the Northern (top) and Southern Hemisphere (bottom). Bias values near zero mean full coverage while values close to 1 indicate a very restricted range of sensor direction.

large circular area well beyond $70^{\circ}$ and the bias is still below 0.8 at the outer border $\left(60^{\circ}\right)$ of the plot while the values at the Northern Hemisphere rise quickly to values $>0.8$ below about $70^{\circ}$ magnetic latitude and $>0.9$ at $\sim 65^{\circ}$. This is due to the large difference in the offset between geographic and geomagnetic coordinates, mentioned already above, which result in more orbit crossings over a much broader latitudinal range at the Southern Hemisphere.

For the analyses of the subsequent sections, we sort the data with respect to the $8 \mathrm{IMF}$ sectors. This reduces the number of data points per bin. The distribution of data over the sectors is not uniform (cf. Haaland et al., 2007, Fig. 1). There are about 2-3 times more data points in the ecliptical plane (sectors 2 and 5) as for purely northward (sector 0 ) or southward (sector 4) directed IMF, but the coverage is good enough to obtain average wind patterns for each sector constellation. To ensure this, we will use the following thresholds for the neutral wind circulation pattern. We use only bins with a minimum of 31 data points and with azimuthal ranges of the cross-track wind measurements which correspond to bias magnitude values $<0.9$. This will limit the Northern Hemisphere plots by a few degrees, but will practically not affect the Southern ones.

\section{Thermospheric wind pattern}

Figure 4 shows the transpolar wind circulation for the Northern Hemisphere in dependence of the IMF clock angle. The data were sorted into 8 separate sectors with $45^{\circ}$ width each centered around IMF clock angles of $0^{\circ}\left(B_{z}^{\mathrm{IMF}}+\right), 45^{\circ}$ $\left(B_{z}^{\mathrm{IMF}}+/ B_{y}^{\mathrm{IMF}}+\right), 90^{\circ}\left(B_{y}^{\mathrm{IMF}}+\right)$ and so forth in the same manner as it was done for the magnetospheric convection analysis with EDI Cluster data in the previous paper of Haaland et al. (2007). The individual panels show the 2-D wind vectors in geomagnetic latitude versus MLT dials with the outer circle at $60^{\circ}$ magnetic latitude. Midday is on top of each dial, midnight at the bottom, dawn to the right, and dusk to the left. The wind vectors are shown as blue lines with the origin at the center of the corresponding bin and indicating the direction and magnitude of the wind at this location by the direction and length of the line, respectively. The length unit is shown in the center area where the dial of the IMF clock angle direction in the GSM y-z-plane is indicated as well; each sector is labeled additionally with the main IMF component, around which this sector is centered.

Figure 5 shows the same presentation for the Southern Hemisphere transpolar wind circulation. The viewing perspective on the plot's dials is as for the Northern Hemisphere, i.e. we are looking at the circulation pattern from above as through a transparent Earth. The patterns are essential similar to the Northern Hemisphere, but they fill nearly the whole viewing area (because of the shallower bias magnitude distribution, see previous section) and the main features of the circulation appear essentially mirror-symmetric with respect to $B_{y}^{\mathrm{IMF}}$.

The ordering of the high-latitude neutral wind circulation in geomagnetic coordinates and its sorting with respect to IMF clock angle in Figs. 4 and 5 reveal some regular patterns that follow to a certain degree the well-known high-latitude 

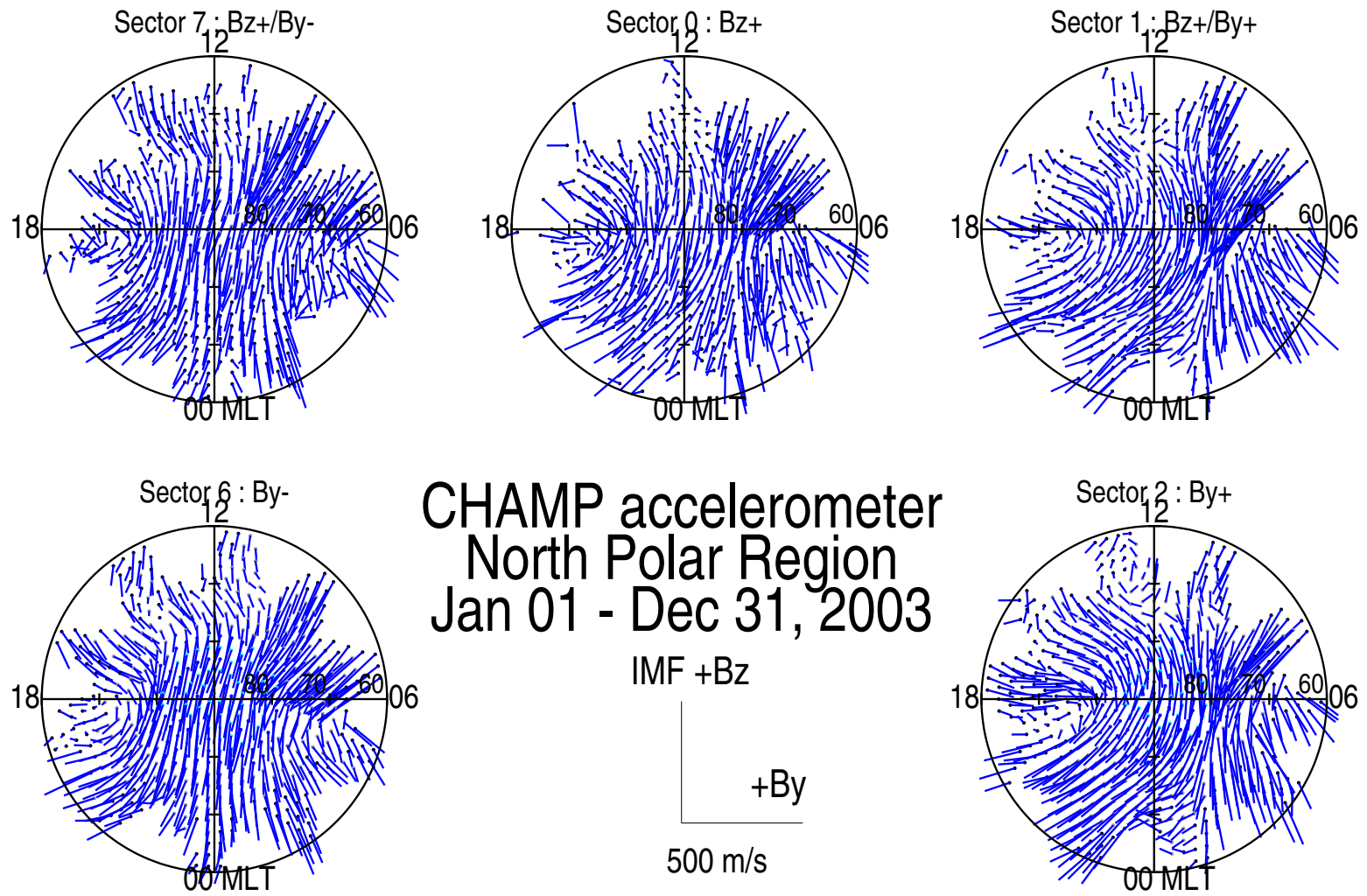

CHAMP accelerometer
North Polar Region
Jan 01 - Dec 31,2003

$\mathrm{IMF}+\mathrm{Bz}$
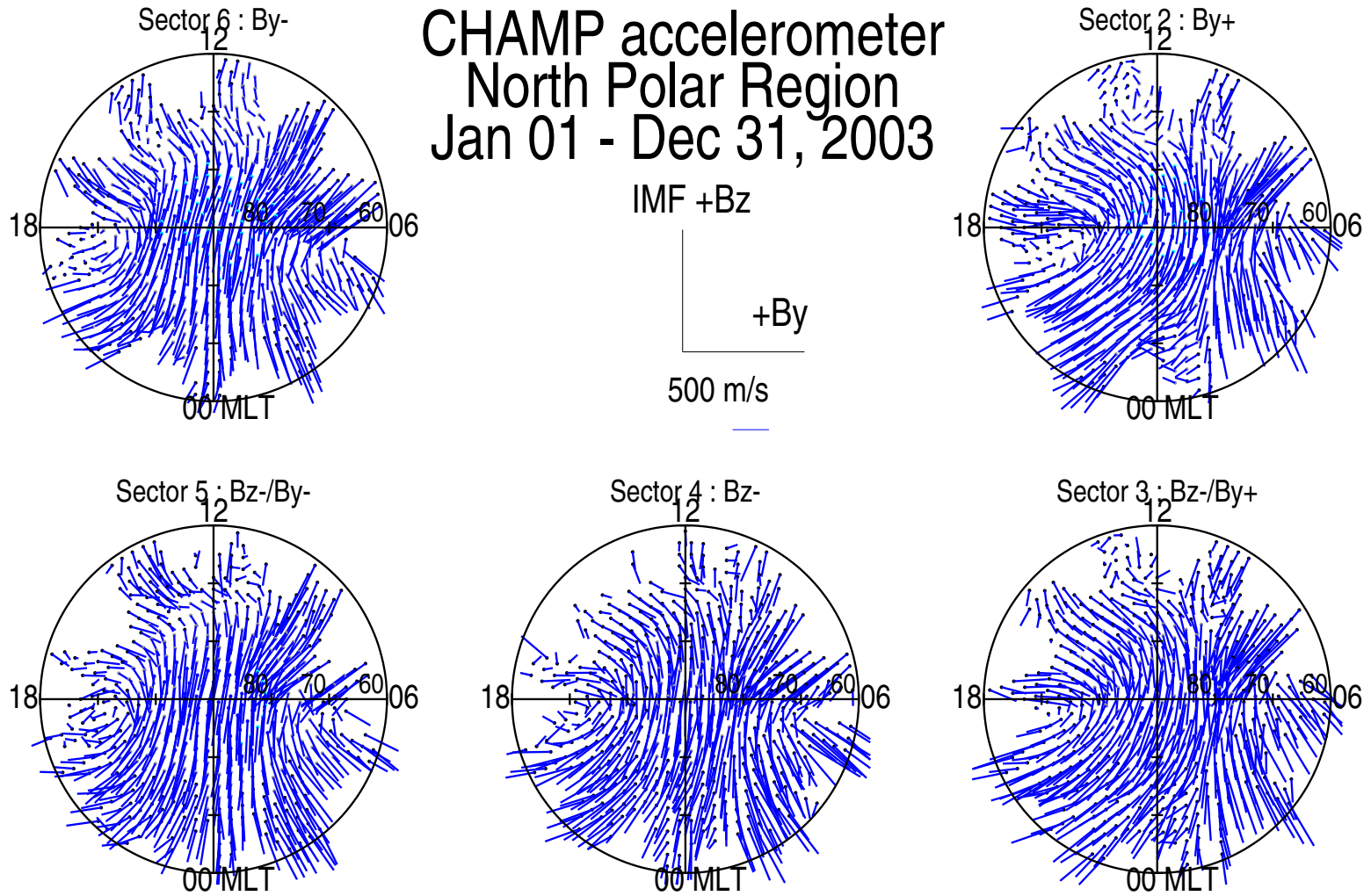

Fig. 4. Thermospheric wind pattern of the North Hemisphere, sorted for the eight IMF sectors. Each dial shows the 2-D wind vectors pattern for the IMF conditions of the sector's clock angle range in geomagnetic coordinates as magnetic latitude versus MLT plot. The outer circle is at $60^{\circ}$ magnetic latitude with the MLT hour labels. The length unit of the wind vectors, shown as blue lines with the origin at the center of each bin is shown in the center area of the plot together with the IMF directions in the GSM y-z-plane.

magnetospheric convection pattern (see, e.g. Ruohoniemi and Greenwald, 2005; Haaland et al., 2007).

The most prominent feature might be the large-scale dusk cell with its focus between $70^{\circ}$ and $75^{\circ}$ magnetic latitude at 18:00 MLT which dominates the high-latitude wind pattern for $B_{y}^{\mathrm{IMF}}+$ (sector 2) at the Northern Hemisphere (Fig. 4) and for $B_{y}^{\mathrm{IMF}}-$ (sector 5) at the Southern (Fig. 5). There are gradual differences in the formation of this clockwise vortex with the turning of the clock angle from positive $B_{z}^{\mathrm{IMF}}$ to negative. It is noticeable already for sector 1 (7) and intensifies toward sector 2 (6) and 3 (5) at the Northern (Southern) Hemisphere, and decays then rapidly toward a curl free (laminar) cross-polar cap streaming from the dayside to the nightside for Southward $B_{z}^{\mathrm{IMF}}-$ of sector 4 and beyond for opposite $B_{y}^{\mathrm{IMF}}$ conditions in sectors 5-7 (3-1) while the focus of the dusk cell remains as a medium-scale feature at about the same location. The potential foci of the magnetospheric plasma convection, on the other hand, are moving 



Fig. 5. Thermospheric wind pattern of the South Hemisphere, sorted for the eight IMF sectors. The same presentation as in Fig. 4, but for the South Hemisphere.

about considerably in dependence on the IMF clock angle (cf. Figs. 7 and 8 in Haaland et al., 2007). The connection line between the positive and negative potential peak on the dawn and dusk side, respectively, makes nearly a full 180deg turn in anticlockwise (clockwise) direction at the Northern (Southern) hemisphere with increasing IMF clock angle. This is also documented in the Tables 1 and 2 below with the average plasma drift azimuth (the $\alpha_{i}$-values, 10th column) obtained from the EDI drift measurements in 2003.

The curl free (laminar) cross-polar cap thermospheric wind streaming of the CHAMP observations is slightly in- clined toward dusk at the Northern Hemisphere and even more clockwise inclined at the Southern. At lower latitudes, these streamlines turn further duskward in the dusk to midnight MLT range and can even reach a slight westward component.

The dawn convection cell does not have such a strong influence on the neutral wind circulation, although some influence is visible in the post-midnight to dawn range at magnetic latitudes of about $75^{\circ}$ and lower. The cross-polar cap streaming is deflected there counter-clockwise so that a radially outward flow appears for apparently all sectors. One 
Table 1. Averaged characteristics of the neutral thermospheric wind (columns 3-7) observed with CHAMP at magnetic latitudes $>80^{\circ}$ of the Northern Hemisphere, compared with averaged magnetospheric plasma drift values (columns 8-12) from Cluster EDI observations of the same time interval in 2003, 1 January-31 December, which were mapped into the ionosphere using the assumption of equipotentiality. Columns 3-7 show the thermospheric wind magnitude $|V|$, its standard deviation $\sigma(|V|)$, the azimuth $\alpha$ with the mean square deviation $\sigma(\alpha)$, and the average number of data points per bin $\left\langle n>\right.$. Columns 8-12 show the corresponding plasma drift magnitude $\left|V_{i}\right|$ and direction $\alpha_{i}$ values and the number of 1-min averages used for each sector and for the global average (bottom line). Some particular values in the table are highlighted to guide the reader; this concerns maximum wind and drift speeds, azimuths closest to the noon-midnight meridian, and minimum angular scatter.

\begin{tabular}{|c|c|c|c|c|c|c|c|c|c|c|c|}
\hline \multicolumn{2}{|c|}{ North Hemisphere } & \multicolumn{5}{|c|}{ CHAMP data } & \multicolumn{5}{|c|}{ Cluster EDI data } \\
\hline Sector & $\begin{array}{l}\text { IMF } \\
\text { direction }\end{array}$ & $\begin{array}{c}|V| \\
{[\mathrm{m} / \mathrm{s}]}\end{array}$ & $\begin{array}{c}\sigma(|V|) \\
{[\mathrm{m} / \mathrm{s}]}\end{array}$ & $\begin{array}{c}\alpha \\
{[\mathrm{deg}]}\end{array}$ & $\begin{array}{l}\sigma(\alpha) \\
{[\mathrm{deg}]}\end{array}$ & $\begin{array}{c}<n> \\
\#\end{array}$ & $\begin{array}{c}\left|V_{i}\right| \\
{[\mathrm{m} / \mathrm{s}]}\end{array}$ & $\begin{array}{c}\sigma\left(\left|V_{i}\right|\right) \\
{[\mathrm{m} / \mathrm{s}]}\end{array}$ & $\begin{array}{r}\alpha_{i} \\
{[\mathrm{deg}]}\end{array}$ & $\begin{array}{r}\sigma\left(\alpha_{i}\right) \\
{[\mathrm{deg}]}\end{array}$ & $\begin{array}{l}n \\
\#\end{array}$ \\
\hline 0 & $B_{z}+$ & 427 & 187 & 167.6 & 9.6 & 131 & 719 & 651 & 31.0 & 98.3 & 940 \\
\hline 1 & $B_{z}+/ B_{y}+$ & 406 & 193 & 166.0 & 18.0 & 224 & 581 & 546 & 115.5 & 78.7 & 2200 \\
\hline 2 & $B_{y}+$ & 464 & 186 & 164.0 & 18.4 & 299 & 541 & 411 & 136.2 & 62.7 & 2758 \\
\hline 3 & $B_{z}-/ B_{y}+$ & 531 & 182 & 164.5 & 13.7 & 188 & 632 & 276 & 151.6 & 30.3 & 1236 \\
\hline 4 & $B_{z}-$ & 567 & 182 & 171.1 & 8.2 & 122 & 791 & 386 & 171.4 & 23.5 & 777 \\
\hline 5 & $B_{z}-/ B_{y}-$ & 607 & 191 & 172.4 & 4.5 & 242 & 622 & 282 & -164.6 & 28.0 & 2068 \\
\hline 6 & $B_{y}-$ & 551 & 190 & 169.1 & 5.1 & 327 & 528 & 304 & -151.4 & 56.0 & 3083 \\
\hline 7 & $B_{z}+/ B_{y}-$ & 494 & 182 & 168.8 & 5.5 & 180 & 483 & 410 & 58.7 & 105.0 & 2558 \\
\hline \multicolumn{2}{|c|}{ all together (average) } & 505 & 209 & 168.2 & 6.8 & 1718 & 576 & 416 & 171.6 & 77.8 & 15620 \\
\hline
\end{tabular}

Table 2. The same as in Table 1, but with respect to the Southern Hemisphere.

\begin{tabular}{|c|c|c|c|c|c|c|c|c|c|c|c|}
\hline \multicolumn{2}{|c|}{ South Hemisphere } & \multicolumn{5}{|c|}{ CHAMP data } & \multicolumn{5}{|c|}{ Cluster EDI data } \\
\hline Sector & $\begin{array}{l}\text { IMF } \\
\text { direction }\end{array}$ & $\begin{array}{c}|V| \\
{[\mathrm{m} / \mathrm{s}]}\end{array}$ & $\begin{array}{c}\sigma(|V|) \\
{[\mathrm{m} / \mathrm{s}]}\end{array}$ & $\begin{array}{c}\alpha \\
{[\mathrm{deg}]}\end{array}$ & $\begin{array}{l}\sigma(\alpha) \\
{[\operatorname{deg}]}\end{array}$ & $\begin{array}{c}<n> \\
\#\end{array}$ & $\begin{array}{c}\left|V_{i}\right| \\
{[\mathrm{m} / \mathrm{s}]}\end{array}$ & $\begin{array}{c}\sigma\left(\left|V_{i}\right|\right) \\
{[\mathrm{m} / \mathrm{s}]}\end{array}$ & $\begin{array}{r}\alpha_{i} \\
{[\mathrm{deg}]}\end{array}$ & $\begin{array}{r}\sigma\left(\alpha_{i}\right) \\
{[\mathrm{deg}]}\end{array}$ & $\begin{array}{l}n \\
\#\end{array}$ \\
\hline 0 & $B_{z}+$ & 388 & 235 & 162.3 & 15.2 & 54 & 381 & 306 & -55.4 & 96.4 & 747 \\
\hline 1 & $B_{z}+/ B_{y}+$ & 449 & 266 & 158.2 & 8.1 & 104 & 437 & 374 & -145.9 & 88.7 & 1759 \\
\hline 2 & $B_{y}+$ & 570 & 264 & 160.1 & 6.6 & 128 & 465 & 347 & -173.7 & 67.6 & 4398 \\
\hline 3 & $B_{z}-/ B_{y}+$ & 592 & 228 & 160.5 & 6.9 & 85 & 553 & 313 & -179.7 & 40.0 & 2857 \\
\hline 4 & $B_{z}-$ & 565 & 234 & 166.0 & 10.6 & 56 & 645 & 334 & 168.2 & 30.8 & 1489 \\
\hline 5 & $B_{z}-/ B_{y}-$ & 528 & 231 & 169.9 & 16.5 & 98 & 562 & 272 & 156.4 & 40.0 & 4357 \\
\hline 6 & $B_{y}-$ & 473 & 231 & 167.9 & 20.1 & 144 & 507 & 321 & 138.4 & 61.6 & 4302 \\
\hline 7 & $B_{z}+/ B_{y}-$ & 454 & 220 & 166.5 & 14.2 & 92 & 566 & 475 & 72.0 & 85.8 & 2059 \\
\hline \multicolumn{2}{|c|}{ all together (average) } & 498 & 265 & 163.7 & 7.5 & 765 & 520 & 344 & 164.5 & 68.7 & 21968 \\
\hline
\end{tabular}

exception might be sector 0 , where the flow pattern is generally less structured with smaller wind amplitudes.

The largest wind amplitudes at high latitudes are usually achieved within the dawn to pre-noon MLT range where the flow is mainly directed toward the pole. The wind amplitudes are still high over the central polar cap area $\left(>80^{\circ}\right)$, pointing predominantly in antisunward direction. At the opposite side, in the dusk to pre-midnight range, also larger wind magnitudes are observed with mostly stronger duskward than antisunward components.

In the region equatorward of the expected cusp location on the dayside near 12:00 MLT and magnetic latitudes from about $70^{\circ}$ down to about $65^{\circ}$, stagnation flows can be noticed, the position of which seems to be related to the $B_{y}^{\mathrm{IMF}}$ component. There are indications of a slight shift in local time (by a small amount of the order of $1 \mathrm{~h}$ ) toward dusk for $B_{y}^{\mathrm{IMF}}+\left(B_{y}^{\mathrm{IMF}}-\right)$ at the Northern (Southern) Hemisphere, respectively, and toward the other side for the opposite $B_{y}^{\mathrm{IMF}}$ polarity. This phenomenon and its relation to the cusp has to be investigated in more detail in a future study that is based on a larger data set with simultaneous observations of several satellites, because its appearence close to the border of the visible area of this study with CHAMP data only is not very obvious, in particular for the Northern Hemisphere (Fig. 4).

The best coverage is available for the central circumpolar area. This region corresponds usually to the central polar cap region of the magnetospheric configuration with 'open' flux tubes convecting from the dayside reconnection region in antisolar direction toward the nightside, modulated mainly by the $B_{y}^{\mathrm{IMF}}$ component in a regular manner (Haaland et al., 2007). In a similar manner as in the study of Förster 
et al. (2007) on high-latitude plasma convection, we averaged therefore in Tables 1 and 2 some transpolar flow characteristics within a circular magnetic latitude range from $80^{\circ}$ to the magnetic pole for the Northern and Southern Hemisphere, respectively. The tables show both the averaged thermospheric wind parameters (columns 3-7) and the plasma convection (columns 8-12) deduced from EDI Cluster measurements for the same time interval in 2003 (cf. also Haaland et al., 2007; Förster et al., 2007, for a larger time interval of this data set). This allows a direct comparison of the flow characteristics of the neutral and ionized components in dependence of the IMF clock angle.

Tables 1 and 2 list in particular the averaged wind $|V|$ and plasma drift $\left|V_{i}\right|$ magnitudes within this circumpolar region, their standard deviations $\sigma(|V|)$ and $\sigma\left(\left|V_{i}\right|\right)$, the average direction azimuths $\alpha$ and $\alpha_{i}$, and their standard deviation $\sigma(\alpha)$ and $\sigma\left(\alpha_{i}\right)$ for the neutral wind and plasma, respectively. The average number of data points per bin $<n>$ and the total number of 1-min data points of EDI measurements are given for all eight IMF clock angle sectors as well as for the summary parameter of the whole data set (as shown in Fig. 1) in the bottom lines.

Tables 1 and 2 reveal a systematic variation of the wind patterns in dependence of the IMF clock angle with some characteristic differences between North and South. The wind amplitudes are smallest for northward IMF and increase steadily with southward turning $B_{z}^{\mathrm{IMF}}$. At the Northern Hemisphere, the maximum amplitude is achieved for sector $5\left(B_{z}^{\mathrm{IMF}}-/ B_{y}^{\mathrm{IMF}}-\right)$, while at the Southern Hemisphere this is the case for sector $3\left(B_{z}^{\mathrm{IMF}}-/ B_{y}^{\mathrm{IMF}}+\right)$. Moreover, for all three sectors with $B_{y}^{\mathrm{IMF}}+$ (sectors 1-3), the average neutral wind amplitudes at the Southern Hemisphere are significantly $(10 \%-20 \%)$ larger than at North, while the opposite is true for $B_{y}^{\mathrm{IMF}}-$ in sectors 5-7. The total average of the wind amplitudes is about the same at the Northern Hemisphere compared with the Southern, while the variances differ significantly. Expressed as standard deviation, they have about a quarter (between 20\% and 40\%) larger values at South with respect to North.

The plasma drift amplitudes are mostly larger than the corresponding thermospheric wind magnitudes for the same IMF sector and there is a slight North-South difference of the averaged drift amplitudes in favour of the Northern Hemisphere as already noted for the more extended EDI data set of 2001-2006 in Förster et al. (2007, by about 7\% there). Remarkably large amplitudes of a secondary maximum in $V_{i}$ can be noted for sector 0 of the North Hemisphere and correspondingly for sector 7 at South, the reason of which is not known. The more extended data set in Förster et al. (2007) does not show this, so that this appears as a particular phenomenon of the geomagnetically active year 2003 . The largest plasma drift amplitudes are seen in sector 4 for purely southward $B_{z}^{\mathrm{IMF}}$ as expected because of most intense dayside reconnection processes for this IMF orientation. The stan- dard deviations of the plasma drift magnitude and drift direction are very large; they are even comparable with the drift amplitude for northward $B_{z}^{\mathrm{IMF}}$ and about half of their magnitude and more ordered for southward $B_{z}^{\mathrm{IMF}}$.

The average direction of the transpolar thermospheric neutral wind flow is about $10^{\circ}$ to $15^{\circ}$ off the noon-midnight direction toward dusk for the Northern Hemisphere and has a generally stronger deflection up to more than $20^{\circ}$ toward dusk for the Southern. There is a small systematic variation of the flow direction with the IMF clock angle. It changes only by a few degrees of the order of $8^{\circ}$ at the Northern and $12^{\circ}$ at the Southern Hemisphere. The flow is usually closer to the noon-midnight meridian for $B_{z}^{\mathrm{IMF}}-$ to $B_{y}^{\mathrm{IMF}}-$ (sectors 4-6) and largest for $B_{y}^{\mathrm{IMF}}+$ (sectors 1-3). The magnitude of the thermospheric wind $>80^{\circ}$ according to Tables 1 and 2 appears to be quite large in comparison with the average drift magnitudes. This might be due to some systematic error of the relatively new method of high-resolution accelerometer measurements that need to be calibrated inflight by using the remote tracking of the orbital decay. This calibration relies on improved representations of the gravitational field that are refined step by step using the same kind of accelerometer observations. As mentioned above, the crosstrack wind determinations used in this study are based on the approach developed by Liu et al. (2006). A systematic error could also arrise from the simplifying assumptions of this approach, considering the complicated streaming situation of neutral air past the spacecraft that results in drag and lift forces which are interpreted in terms of density and crosstrack winds. As noted before in Sect. 2.1, the measurement error due to the along-track wind component is largest in the high-latitude regions. The estimated neutral wind orientation, however, is less affected by these sources of error.

The standard deviation of the flow direction obtained from the sample of the flow directions at all bins within the circumpolar region, shows remarkable differences between $B_{y}^{\mathrm{IMF}}+$ and $B_{y}^{\mathrm{IMF}}-$ conditions at the Northern Hemisphere. The flow is much more aligned for the latter, with a minimum value for sector 5, where the wind amplitude maximizes. At the Southern Hemisphere, there is a tendency for the opposite $B_{y}^{\mathrm{IMF}}$ behaviour with smaller values for $B_{y}^{\mathrm{IMF}}+$, but the contrast is not that large there.

We repeated the same analysis of the average thermospheric wind behaviour as in Tables 1 and 2 with various IMF bias vector filterings (as explained in Sect. 2.2) to find out whether larger IMF direction changes in the 30-min running box-car intervals play a role. A threshold of 0.9 resulted in practically the same results as for unfiltered data (not shown), but the statistics became poorer. Solar wind and IMF changes within about $30 \mathrm{~min}$ seem not to make a noticeable change to the average statistical pattern. This analysis should be repeated with a larger data set and various, in particular larger filter lengths. 


\section{Discussion}

We have analyzed the CHAMP cross-track accelerometer measurements of about 5600 transpolar overflights above the high-latitude polar regions recorded between 1 January and 31 December 2003. Statistical patterns of the cross-polar thermospheric circulation were obtained separately for the Northern and Southern Hemispheres and they were analyzed with respect to the direction of the interplanetary magnetic field (IMF clock angle) as measured on ACE. Special care has been taken on the correct estimation of the time shift of the ACE data, when propagating them to the near-Earth space by considering the orientation of the IMF "phase fronts" according to a method invented by Weimer et al. (2003) in the slightly modified version of Haaland et al. (2006).

The importance of the study lies in the direct comparison of the IMF-dependent high latitude thermospheric wind patterns derived from CHAMP cross-track measurements, with ionospheric patterns from the Cluster EDI instrument over the same year of observation (2003). Previous satellite studies where upper atmospheric ion and neutral flows have been compared directly have been mostly limited to case studies. Comparison is also made between the CHAMP measurements at an altitude of $\sim 400 \mathrm{~km}$ and ground-based FPIs observing thermospheric winds at $\sim 250 \mathrm{~km}$ altitude (see below). The results indicate either an unexpected shear over this height range, despite large viscosity, or imply that the derivation of winds needs to be re-investigated.

The transpolar thermospheric neutral wind circulation from the dayside to the nightside is substantially modified by the high-latitude magnetospheric convection pattern. The mapping into geomagnetic coordinates discloses the tracing of larger-scale plasma convection features already for the average pattern that comprise the whole data set (Fig. 1). These features confirm the tight linking of the high-latitude thermosphere circulation pattern to the geomagnetic field configuration and the related electrodynamics but also some characteristic differences between North and South. The main magnetospheric convection structures are already reflected in the average circulation pattern like, e.g. the stagnation region a few degree equatorward to the cusp footpoints at about $65^{\circ}-$ $70^{\circ}$ around noon on the dayside, the large dusk convection cell with the focus at about $70^{\circ}-75^{\circ}$ magnetic latitude close to 18:00 MLT, the duskward deflection in the dusk to midnight sector at the nightside, and the smaller outward neutral wind deflection zone in the region of the dawnside convection cell near $70^{\circ}$ magnetic latitude in the midnight to dawn sector. The hemispheric differences manifest themselves in a slightly stronger deflection toward dusk for the cross-polar circulation within the central part of the Southern polar cap, and broader, more diffuse tracings of the cusp region and of the large dusk convection cell at South.

Coming from the dayside, where the large-scale thermospheric circulation is initiated by the dominating EUV heating source, the thermospheric neutral wind is subject to vari- ous forcings during its transpolar flow at high latitudes. The first "obstacle" on its way is the cusp region near noon at about $70^{\circ}$ magnetic latitude, where a considerable air upwelling due to particle and Joule heating was observed to create a persistent medium-scale local stagnation region of the horizontal flow, as shown in previous studies of CHAMP data analyses (Lühr et al., 2004; Schlegel et al., 2005). Vertical neutral air movements, i.e. vertical accelerations due to local heating sources below the CHAMP orbit are not considered here. They are an important part of the high-latitude thermospheric dynamics and will be dealt with separately.

Localized heating sources in the cusp region and the auroral zone which are due to particle precipitations and to current closure resulting in Joule heating contribute to the horizontal acceleration of the neutral gas at high latitudes. They are part of the driving forces of the global thermospheric neutral air circulation acting via pressure-gradient forces. But the various terms of the horizontal acceleration at high latitudes comprise mainly the ion drag due to forcings of the imprinted magnetospheric plasma convection as well as the Coriolis and centrifugal forces of the rotating Earth (FullerRowell and Rees, 1984). They are part of the equation of motion for the neutral particles, where they are in dynamic balance with the pressure-gradient and viscous forces as well as with the non-linear momentum advection term (cf., e.g. Killeen and Roble, 1984, Appendix).

The Coriolis and centrifugal forces are related to an Earthfixed geographic coordinate system with the angular rotation moment of Earth $\omega=2 \pi / 86164 \boldsymbol{e}_{z}^{\text {geoc }}\left[\mathrm{s}^{-1}\right]$ aligned with the Earth's rotation axis. A numerical description of the thermospheric motion in the geomagnetic frames of Figs. 1, 4, and 5 would firstly require the performance of a (hypothetical) coordinate transformation of these two terms into the common geomagnetic coordinate system. This transformation would be small for the Northern Hemisphere (about $8^{\circ}$ ), but it is considerable for the Southern Hemisphere, where the distance between the geographic and geomagnetic axes amounts to $25^{\circ}$. This should be kept in mind for the subsequent data interpretations.

By sorting the data into 8 disjunct clock angle ranges (sectors), we show the gradual differences of the transpolar circulation pattern for different IMF clock angle ranges. This is illustrated in Figs. 4 and 5 for the thermospheric wind pattern over the high-latitude thermosphere from the pole down to $60^{\circ}$ magnetic latitude at the outer border of the plot's dials as well as in the Tables 1 and 2 for averages of some characteristic neutral wind and plasma drift parameters in the central polar cap region $>80^{\circ}$ magnetic latitude. It should be noted that the magnitudes of the wind speeds derived here from the CHAMP accelerometer measurements appear to be quite large, in particular when comparing them with the EDI Cluster observations. They amount to about $80-90 \%$ of the average ion drift velocities. Considering the fact, that the ion drift serves as driving force for the neutrals and that the neutral component needs a certain response time to the highly 
variable magnetospheric convection pattern that range from tens of minutes to hours, this seems to be somehow too large.

The comparison with recent comprehensive compilations of high-latitude ground-based FPI neutral wind observations at both hemispheres by, e.g. Emmert et al. (2006a,b), show that the CHAMP winds are a factor 2-3 larger than the ground-based observations. FPI measurements are, however, essentially taken from a different altitude range $(\sim 250 \mathrm{~km})$ and they are usually integrating over a larger altitude range. The CHAMP satellite measurements, on the other hand, are in-situ observations from F2-layer altitudes near $400 \mathrm{~km}$ and represent upper thermosphere conditions. Comparable wind measurements from this altitude range are relatively seldom for the last two decades. In-situ thermospheric wind measurements were obtained in the 1970s and early 1980s by the AE-C and DE-2 satellites. Their published measured wind amplitudes are comparable with the magnitudes obtained in this study as, e.g. in the papers of Thayer et al. (1987, Fig. 3) and Thayer and Killeen (1993, Table 1), where DE-2 observations of a high solar activity period are summarized explicitly for the polar cap with magnetic latitudes $>80^{\circ}$ (as in our study) with mean wind speeds between $400 \mathrm{~m} / \mathrm{s}$ and $600 \mathrm{~m} / \mathrm{s}$, depending on geomagnetic activity and on the hemisphere. Further, Killeen et al. (1995) reported about FPI measurements in Thule and Sondre Stromfjord and their solar cycle, geomagnetic activity and IMF dependencies. Their summary representations in Fig. 8 suggests wind amplitudes close to $400 \mathrm{~m} / \mathrm{s}$ for comparable conditions for the Thule FPI.

The large dusk cell neutral wind circulation dominates for $B_{y}^{\mathrm{IMF}}+$ conditions at the Northern Hemisphere in sectors 1 to 3 (Fig. 4) and it exists mirror-symmetric for $B_{y}^{\mathrm{IMF}}-$ at the Southern Hemisphere also as a large dusk convection cell in sectors 7 to 5 (Fig. 5). The corresponding magnetospheric convection pattern are known as large, round-shaped convection cells as shown, e.g. in the studies of Ruohoniemi and Greenwald (2005) and Haaland et al. (2007). According to the above mentioned superposition of Coriolis and centrifugal forces, the plasma convection favours this clockwise circulation vortex of the neutral air at the dusk side. A large-scale cyclonic vortex appears over the major part of the high latitude thermospheric circulation. It has its focal point near $18 \mathrm{MLT}$ at $70^{\circ}-75^{\circ}$ magnetic latitude and comprises the whole dusk side high-latitude region all the way from postnoon to pre-midnight MLT hours and over the pole down to about $80^{\circ}-75^{\circ}$ toward the dawn side. The smaller, "crescentshaped" dawn side convection cell is suppressed in its imprint on the neutral air motion due to the opposite action of Coriolis and centrifugal forces on its poleward side; at the equatorward, subauroral side it might contribute to a faster outward flow, if the movement has an eastward component there.

For purely southward $B_{z}^{\mathrm{IMF}}$ (sector 4), the dawn and dusk cells become more symmetric and this gives the way for a curl free, laminar neutral wind circulation closer to the noon- midnight meridian. The transpolar flow in the central polar cap becomes deflected toward dusk because of the Coriolis force. The westward deflection of the Coriolis force in the midnight and dusk sector entrains the gas parcel to build up momentum through ion drag. In the dawn sector, the gas parcel tends to be pushed out of the dawn anticlockwise circulation by the Coriolis force. The angle of deflection from the noon-midnight meridian is smallest for sector 5 at both hemispheres, i.e. close to those conditions where the driving transpolar magnetospheric convection has its largest magnitudes (cf. Tables 1 and 2).

In the sectors with opposite $B_{y}^{\mathrm{IMF}}$ polarity, i.e. sectors 5 to 7 in the Northern and sectors 3 to 1 in the Southern Hemisphere, the dusk cell is still present, but to a much smaller extent. The round-shaped magnetospheric convection cells which are known from the plasma potential pattern to exist at the dawn side, cannot have a corresponding imprint on the neutral air circulation due to the unfavourable action of the Coriolis and centrifugal forces, as mentioned above. The transpolar thermospheric wind flow is curl free in a wider latitudinal range and is directed from the pre-noon hours to pre-midnight, forming an angle of about $10^{\circ}-15^{\circ}$ with the noon-midnight meridian at the Northern and more than $20^{\circ}$ at the Southern Hemisphere. The non-divergent, laminar flow within the central polar cap under the latter conditions is also manifested in the Tables 1 and 2 by smaller average angular variances (6th column of the tables) for these sectors. Interestingly, the comparison of the $B_{y}^{\mathrm{IMF}}$ dependency of FPI neutral wind pattern obtained at $\sim 250 \mathrm{~km}$ altitude (by, e.g. Emmert et al., 2006b, Fig. 8) with the CHAMP wind pattern seems to show a systematic clockwise shift in the wind direction with increasing height. The CHAMP vectors appear to be rotated by $\sim 30^{\circ}-40^{\circ}$ with respect to the FPI observations. The latter measurements, however, were done during quiet geomagnetic conditions near the winter solstice, so that definitive conclusions about a directional shear cannot be drawn yet. This subject needs further investigations in a separate study.

The largest wind amplitudes are gained for sector 5 at North and sector 3 at South, i.e. not for purely southward $B_{z}^{\mathrm{IMF}}$ conditions, where the magnetospheric convection speed maximizes (8th column, see also Haaland et al., 2007, Table 3), but rather for those sectors where the antisunward magnetospheric convection in the central polar cap is in favour of a curl free, laminar flow (i.e. small values of $\sigma(\alpha)$ in column 6), closely aligned with the noon-midnight meridian at the Southern Hemisphere and with a slightly duskward plasma flow at the Northern. The large skewing of the magnetospheric plasma convection pattern towards dawn (dusk) for $B_{y}^{\mathrm{IMF}}+\left(B_{y}^{\mathrm{IMF}}-\right)$ at the Northern Hemisphere and oppositely at the Southern Hemisphere is not reflected in the neutral wind cross-polar circulation pattern. It is rather the formation of the large round-shaped plasma convection cell which is present under $B_{y}^{\mathrm{IMF}}+\left(B_{y}^{\mathrm{IMF}}-\right)$ conditions at the Northern (Southern) Hemisphere at the dusk side which 
traces in the thermospheric wind pattern and which apparently act as an obstacle for the neutral wind flow across the polar region at high latitudes. The curl free noon-midnight aligned plasma flow seems on the contrary to operate as a "pressure valve", allowing largest cross-polar thermospheric wind amplitudes for IMF angle ranges corresponding to sector 5 at North and sector 3 at South.

The neutral wind circulation pattern forms the background movement for the forcing magnetospheric plasma movement and acts as a "drag" on convection (Cole, 1963; Hill, 1976). The neutral wind and plasma forces interact and adjust each other within characteristic time scales of the order of tens of minutes to hours (depending on plasma density). The coupling must therefore have some influence on the amplitudes of the magnetospheric circulation pattern. This could be the reason for the observed braking of the mirror-symmetry for positive and negative $B_{y}^{\mathrm{IMF}}$ at the Northern and Southern Hemisphere (cf. Haaland et al., 2007; Förster et al., 2007).

For purely northward $B_{z}^{\mathrm{IMF}}+$, we observe the smallest cross-polar thermospheric neutral wind amplitudes (see Tables 1 and 2) and the neutral wind pattern appears to be less ordered (see Figs. 4 and 5, sector 0). The four-cell pattern with sunward plasma movement between the so-called lobe cells at higher latitudes on the dayside and the relatively small main cells are forcing the neutral wind in opposite direction within a relatively small area. The plasma convection has its largest variability in amplitude and direction even for this IMF clock angles. The thermospheric wind pattern for $B_{z}^{\mathrm{IMF}}+$ is therefore not favourable for a well-ordered crosspolar neutral wind circulation.

The time interval of the full year 2003 was chosen to get an optimal seasonal coverage of the high-latitude regions of both hemispheres. Different illumination conditions have an influence on the magnetospheric plasma convection pattern. Higher ionospheric conductances lead to an attenuation of the electric field in the ionosphere and therefore to smaller convection velocities. The analysis of seasonal differences was the focus of a previous paper of Lühr et al. (2007) on CHAMP accelerometer measurements for high-latitude thermospheric wind analysis, which uses about one third of the interval of this study centered closely around the June solstice. They notice a clearly weaker cross-polar cap flow at the Southern (winter) Hemisphere, assuming that the ion drag is smaller at the winter hemisphere with on average smaller ion/neutral collision frequencies due to lower plasma densities at the dark polar cap. This should be checked in a future study by a correlation analysis with the plasma density measurements obtained with the planar Langmuir probe onboard CHAMP at the same time.

In this study, we observe hemispheric differences with respect to significantly enhanced standard deviations of the neutral wind amplitudes and smaller average plasma drift magnitudes (cf. Tables 1 and 2) at the Southern Hemisphere while the mean neutral wind amplitudes are about the same. The considerable larger offset between geographic and geo- magnetic coordinates (see Sect. 3) that allowed on the one hand a better applicability of the method over a wider latitudinal range at the Southern Hemisphere, means on the other hand a larger daily "wobbling" of the polar cap area within the geographic frame. A part of the forces that the neutral air is subjected relates clearly to geographic coordinates like the Coriolis and the centrifugal forces as well as the dayside heating source by EUV radiation. Another part, the electrodynamic forces, are bound to geomagnetic coordinates (Hays et al., 1984; Thayer et al., 1987; Richmond et al., 2003) and this aspect was the motivation for the coordinates selected for this study. The neutral air parcels have to obey both forcings and their resulting movement is the sum and (probably nonlinear) adjustment of them to each other. The larger displacement of the geographic and geomagnetic poles at the South might be the reason for a wider range of counteracting forces with time scales that might be close to each other. This could finally have resulted in the larger variances of the Southern neutral wind pattern observed.

In any case, this subject needs further studies with larger data sets that have a better, more uniform seasonal coverage. Larger data sets will be available soon from the CHAMP itself, but also from other running and forthcoming near-Earth missions. A larger statitical study will also be useful and necessary to study further dependencies of the high-latitude thermospheric wind pattern on, e.g. solar radiation flux related to $F_{10.7}$, and other IMF and solar wind parameters. A larger number of quasi simultaneous measurements by several nearEarth spacecraft will allow to solve the problem of the missing along-track neutral wind component that is needed to enhance the precision of the cross-track measurement as well. One could overcome the deficiency of the method by gathering data for the other components from other satellites at different orbits that come close in space and time.

\section{Conclusions}

Summarizing the main findings of this study, we conclude the following.

- Large-scale clockwise circulation vortices at the dusk side appear as a dominant feature of the high-latitude cross-polar thermospheric wind pattern for $B_{y}^{\mathrm{IMF}}+$ at the Northern, and $B_{y}^{\mathrm{IMF}}-$ at the Southern Hemisphere. This corresponds to the appearance of the large roundshaped dusk cells of the plasma convection pattern under these conditions.

- The largest magnitudes of the cross-polar upper thermospheric winds over the central polar cap occur un$\operatorname{der} B_{z}^{\mathrm{IMF}}-/ B_{y}^{\mathrm{IMF}}-($ sector 5) conditions at the Northern Hemisphere, but under $B_{z}^{\mathrm{IMF}}-/ B_{y}^{\mathrm{IMF}}+$ (sector 3) conditions at the Southern. Moreover, the solar wind induced magnetospheric ion drift pattern controls the neutral wind speed, allowing larger wind amplitudes 
for $B_{y}^{\mathrm{IMF}}$ - values (sectors 5-7) at the Northern and for $B_{y}^{\mathrm{IMF}}+$ values (sectors $1-3$ ) at the Southern Hemisphere. The momentum transfer from the ions to the neutrals has therefore been shown to be asymmetric at the opposite hemispheres in dependence of $B_{y}^{\mathrm{IMF}}$.

- The dawn convection cell is not favoured for the thermospheric wind response due to the counteracting Coriolis and centrifugal forces in this region. It degenerates to a disturbance zone at $\sim 70^{\circ}$ with equatorward flow deflections at lower latitudes.

- Despite the large dusk cell circulation under certain circumstances, the thermospheric cross-polar circulation is only moderately affected by small azimuth deflections of the main flow direction within the central polar cap region in contrast to the systematic rotation of the convection streamlines of the ionized component. The small deflections show a regular dependence on the IMF clock angle with larger deviations from the noon-midnight meridian toward dusk for $B_{y}^{\mathrm{IMF}}+$ than for $B_{y}^{\mathrm{IMF}}$ - and systematically larger deflections at the Southern compared with the Northern Hemisphere.

- There are significant differences between the Northern and Southern Hemisphere in the variance of the neutral wind. At South we note about 25\% larger standard deviations and the circulation appears less structured in comparison with the Northern Hemisphere. This might be explained by the "stirring" effect due to the larger geographic-geomagnetic offset, because the neutral gas has to obey both geographically and geomagnetically oriented forcings. It might partly be due to season/solar illumination differences between the hemispheres. The larger variance might have consequences for different thermospheric heating rates at the opposite high latitude regions.

Acknowledgements. Work at GeoForschungsZentrum Potsdam (M. Förster and S. Rentz) was supported by Deutsche Forschungsgemeinschaft (DFG). Research at the University of Bergen was supported by the Norwegian Research Council. We thank the ACE SWEPAM and MAG instrument teams and the ACE Science Center for providing the ACE data.

Topical Editor I. A. Daglis thanks two anonymous referees for their help in evaluating this paper.

\section{References}

Aruliah, A. L., Farmer, A. D., Rees, D., and Brändström, U.: The seasonal behavior of high-latitude thermospheric winds and ion velocities observed over one solar cycle, J. Geophys. Res., 101, 15 701-15 711, 1996.

Aruliah, A. L., Griffins, E. M., McWhirter, I., et al.: First tristatic studies of meso-scale ion-neutral dynamics and energetics in the high-latitude upper atmosphere using collocated FPIs and EISCAT radar ionosphere, Geophys. Res. Lett., 31, L03802, doi: 10.1029/2003GL018469, 2004.

Banks, P. M.: Magnetospheric processes and the behavior of the neutral atmosphere, Space Res., 12, 1051-1067, 1972.

Codrescu, M. V., Fuller-Rowell, T. J., and Foster, J. C.: On the importance of E-field variability for Joule heating in the high-latitude thermosphere, Geophys. Res. Lett., 22, 2393-2396, 1995.

Codrescu, M. V., Fuller-Rowell, T. J., Foster, J. C., Holt, J. M., and Cariglia, S. J.: Electric field variability associated with the Millstone Hill electric field model, J. Geophys. Res., 105, 52655274, 2000.

Cole, K. D.: Damping of magnetospheric motions by the ionosphere, J. Geophys. Res., 68, 3231-3235, 1963.

Coroniti, F. V. and Kennel, C. F.: Can the ionosphere regulate magnetospheric convection?, J. Geophys. Res., 78, 2837-2851, 1973

Crowley, G. and Hackert, C. L.: Quantification of High Latitude Electric Field Variability, Geophys. Res. Lett., 28, 2783-2786, 2001.

Emmert, J. T., Faivre, M. L., Hernandez, G., et al.: Climatologies of nighttime upper thermospheric winds measured by groundbased Fabry-Perot interferometers during geomagnetically quiet conditions: 1. Local time, latitudinal, seasonal, and solar cycle dependence, J. Geophys. Res., 111, A12302, doi:10.1029/ 2006JA011948, 2006a.

Emmert, J. T., Hernandez, G., Jarvis, M. J., Niciejewski, R. J., Sipler, D. P., and Vennerstrom, S.: Climatologies of nighttime upper thermospheric winds measured by ground-based Fabry-Perot interferometers during geomagnetically quiet conditions: 2. High-latitude circulation and interplanetary magnetic field dependence, J. Geophys. Res., 111, A12303, doi: 10.1029/2006JA011949, 2006b.

Forbes, J. M., Roble, R. G., and Marcos, F. A.: Magnetic activity dependence of high-latitude thermospheric winds and densities below 200 km, J. Geophys. Res., 98, 13 693-13 702, 1993.

Förster, M., Paschmann, G., Haaland, S. E., et al.: High-latitude plasma convection from Cluster EDI: Variances and solar wind correlations, Ann. Geophys., 25, 1691-1707, 2007, http://www.ann-geophys.net/25/1691/2007/.

Fuller-Rowell, T. J. and Rees, D.: Interpretation of an anticipated long-lived vortex in the lower thermopshere following simulation of an isolated substorm, Planet. Space Sci., 32, 69-85, 1984.

Griffin, E. M., Aruliah, A., Müller-Wodarg, I. C. F., and Aylward, A.: Comparison of high-latitude thermospheric meridional winds II: combined FPI, radar and model Climatologies, Ann. Geophys., 22, 863-876, 2004a, http://www.ann-geophys.net/22/863/2004/.

Griffin, E. M., Müller-Wodarg, I. C. F., Aruliah, A., and Aylward, A.: Comparison of high-latitude thermospheric meridional winds I: optical and radar experimental comparisons, Ann. Geophys., 22, 849-862, 2004b, http://www.ann-geophys.net/22/849/2004/.

Haaland, S. E., Paschmann, G., and Sonnerup, B. U. Ö.: Comment on "A new interpretation of Weimer et al.'s solar wind propagation delay technique" by Bargatze et al., J. Geophys. Res., 111, A06102, doi:10.1029/2005JA011376, 2006.

Haaland, S. E., Paschmann, G., Förster, M., et al.: High-latitude plasma convection from Cluster EDI measurements: Method and 
IMF-dependence, Ann. Geophys., 25, 239-253, 2007,

http://www.ann-geophys.net/25/239/2007/.

Hays, P. B., Meriwether, J. W., and Roble, R. G.: Nighttime thermospheric winds at high latitudes, J. Geophys. Res., 84, 1905-1913, 1979.

Hays, P. B., Killeen, T. L. N. W. S., Wharton, L. E., et al.: Observations of the dynamics of the polar thermosphere, J. Geophys. Res., 89, 5597-5612, 1984.

Hill, T. W.: Mercury and Mars: The role of ionospheric conductivity in the acceleration of magnetospheric particles, Geophys. Res. Lett., 3, 429-432, 1976.

Killeen, T. L. and Roble, R. G.: An analysis of the high-latitude thermospheric wind pattern calculated by a Thermospheric General Circulation Model 1. Momentum forcing, J. Geophys. Res., 89, 7509-7522, 1984.

Killeen, T. L., Hays, P. B., Carignan, G. R., et al.: Ion-neutral coupling in the high-latitude $\mathrm{F}$ region: Evaluation of ion heating terms from Dynamics Explorer 2, J. Geophys. Res., 89, 74957508, 1984.

Killeen, T. L., Heelis, R. A., Hays, P. B., Spencer, N. W., and Hanson, R. A.: Neutral motions in the polar thermosphere for northward interplanetary magnetic field, Geophys. Res. Lett., 12, 159162,1985

Killeen, T. L., Smith, R. W., Spencer, N. W., et al.: Mean neutral circulation in the winter polar $F$ region, J. Geophys. Res., 91, 1633-1649, 1986.

Killeen, T. L., Won, Y.-I., Niciejewski, R. J., and Burns, A. G.: Upper thermosphere winds and temperatures in the geomagnetic polar cap: Solar cycle, geomagnetic activity, and interplanetary magnetic field dependencies, J. Geophys. Res., 100, $21327-$ $21342,1995$.

Killeen, T. L., Wu, Q., Solomon, S. C., et al.: TIMED Doppler Interferometer: Overview and recent results, J. Geophys. Res., 111, A10S01, doi:10.1029/2005JA011484, 2006.

Liu, H., Lühr, H., Henize, V., and Köhler, W.: Global distribution of the thermospheric total mass density derived from CHAMP, J. Geophys. Res., 110, A04301, doi:10.1029/2004JA010741, 2005.

Liu, H., Lühr, H., Watanabe, S., Köhler, W., Henize, V., and Visser, P.: Zonal winds in the equatorial upper thermosphere: Decomposing the solar flux, geomagnetic activity, and seasonal dependencies, J. Geophys. Res., 111, A07307, doi:10.1029/ 2005JA011415, 2006.

Lühr, H., Rother, M., Köhler, W., Ritter, P., and Grunwaldt, L.: Thermospheric upwelling in the cusp region: Evidence from CHAMP observations, Geophys. Res. Lett., 31, L06805, doi: 10.1029/2003GLL019314, 2004.

Lühr, H., Rentz, S., Ritter, P., Liu, H., and Häusler, K.: Average thermospheric wind pattern over the polar regions, as observed by CHAMP, Ann. Geophys., 25, 1093-1101, 2007, http://www.ann-geophys.net/25/1093/2007/.

Marcos, F. A. and Forbes, J. M.: Thermospheric wind from the satellite electrostatic triaxial accelerometer system, J. Geophys. Res., 90, 6543-6552, 1985.

Matsuo, T., Richmond, A. D., and Hensel, K.: High-latitude ionospheric electric field variability and electric potential derived from DE-2 plasma drift measurements: Dependence on IMF and dipole tilt, J. Geophys. Res., 108, 1005, doi:10.1029/ 2002JA009429, 2003.
McComas, D. J., Bame, S. J., Barker, P., Feldman, W. C., Phillips, J. L., Riley, P., and Griffee, J. W.: Solar Wind Electron Proton Alpha Monitor (SWEPAM) for the Advanced Composition Explorer, Space Sci. Rev., 86, 563-612, 1998.

McCormac, F. G., Killeen, T. L., Gombosi, E., Hays, P. B., and Spencer, N. W.: Configuration of the high-latitude thermosphere neutral circulation for IMF By negative and positive, Geophys. Res. Lett., 12, 155-158, 1985.

Ponthieu, J.-J., Killeen, T. L., Lee, K.-M., Carignan, G. R., Hoegy, W. R., and Brace, L. H.: Ionosphere-thermospheric momentum coupling at solar maximum and solar minimum from DE-2 and AE-C data, Phys. Scripta, 37, 447-454, 1988.

Rees, D., Fuller-Rowell, T. J., Gordon, R., et al.: A theoretical and empirical study of the response of the high latitude thermosphere to the sense of the "Y" component of the interplanetary magnetic field, Planet. Space Sci., 34, 1-40, 1986.

Reigber, C., Lühr, H., and Schwintzer, P.: CHAMP mission status, Adv. Space Res., 30, 129-134, 2002.

Richmond, A. D.: Ionospheric electrodynamics using magnetic Apex coordinates, J. Geomag. Geoelectr., 47, 191-208, 1995.

Richmond, A. D., Lathuillère, C., and Vennerstroem, S.: Winds in the high-latitude lower thermosphere: Dependence on the interplanetary magnetic field, J. Geophys. Res., 108, 1066, doi: 10.1029/2002JA009493, 2003.

Ruohoniemi, J. M. and Baker, K. B.: Large-scale imaging of highlatitude convection with Super Dual Auroral Radar Network HF radar observations, J. Geophys. Res., 103, 20 797-20 811, 1998.

Ruohoniemi, J. M. and Greenwald, R. A.: Dependencies of high-latitude plasma convection: Consideration of interplanetary magnetic field, seasonal, and universal time factors in statistical patterns, J. Geophys. Res., 110, A09204, doi:10.1029/ 2004JA010815, 2005.

Schlegel, K., Lühr, H., St.-Maurice, J.-P., Crowley, G., and Hackert, C.: Thermospheric density structures over the polar regions observed with CHAMP, Ann. Geophys., 23, 1659-1672, 2005, http://www.ann-geophys.net/23/1659/2005/.

Shepherd, G. G., Thuillier, G., Gault, W. A., et al.: WINDII, the WIND imaging interferometer on the Upper Atmosphere Research Satellite, J. Geophys. Res., 98, 10 725-10 750, 1993.

Smith, C. W., L'Heureux, J., Ness, N. F., Acuña, M. H., Burlaga, L. F., and Scheifele, J.: The ACE magnetic fields experiment, Space Sci. Rev., 86, 613-632, 1998.

Thayer, J. P. and Killeen, T. L.: A kinematic analysis of the highlatitude thermospheric neutral circulation pattern, J. Geophys. Res., 98, 11 549-11 565, 1993.

Thayer, J. P., Killeen, T. L., McCormac, F. G., Tschan, C. R., Ponthieu, J.-J., and Spencer, N. W.: Thermospheric neutral wind signatures dependent on the east-west component of the interplanetary magnetic field for Northern and Southern Hemispheres as measured from Dynamics Explorer-2, Ann. Geophys., 5a, 363368, 1987.

Weimer, D. R., Ober, D. M., Maynard, N. C., et al.: Predicting interplanetary magnetic field (IMF) propagation delay times using the minimum variance technique, J. Geophys. Res., 108, 1026, doi:10.1029/2002JA009405, 2003. 